\title{
Choice of nasal nitric oxide technique as first-line test for primary ciliary dyskinesia
}

\author{
J.K. Marthin and K.G. Nielsen
}

\begin{abstract}
Nasal nitric oxide ( $\mathrm{nNO}$ ) has a well-known potential as an indirect discriminative marker between patients with primary ciliary dyskinesia (PCD) and healthy subjects, but real-life experience and usefulness in young children is sparsely reported. Three nNO sampling methods were examined and compared as first-line tests for PCD.

Healthy subjects, confirmed PCDs, consecutive referrals with PCD-like symptoms and patients with cystic fibrosis (CF) had $\mathrm{nNO}$ sampled during breath hold (BH-nNO), oral exhalation against resistance (OE-R-nNO) and tidal breathing (TB-nNO) aiming to expand age range into infancy.

282 subjects, 117 consecutive referrals, 59 PCDs, 49 CF patients and 57 healthy subjects, were included. All methods separated significantly between PCD and non-PCD, including CF with reliability, in ranking order $\mathrm{BH}-\mathrm{nNO}>\mathrm{OE}-\mathrm{R}-\mathrm{nNO}>\mathrm{TB}-\mathrm{nNO}$. Acceptability in children ranked in reverse order. A problematic high fraction (39\%) of false positive TB-nNO was found in young children. An unexpected large fraction (6.8\%) of PCDs had nNO values above cut-off.

nNO is a helpful first-line tool in real-life PCD work-up in all age groups if the sampling method is chosen according to age. nNO can be misleading in a few patients with true PCD. Further studies are strongly needed in young children.
\end{abstract}

KEYWORDS: Children, methods, nasal nitric oxide, primary ciliary dyskinesia

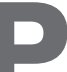

rimary ciliary dyskinesia (PCD) is an inherited disorder characterised by abnormalities in ciliary structure and/or function, impaired mucociliary clearance and recurrent or chronic airway infections [1], which are a serious threat to lung function, probably even in young children [2].

Early diagnosis is important, since lung function may improve or stabilise after diagnosis and initiation of treatment [2], and may be achieved by support from measurement of nasal nitric oxide (nNO) $[3,4]$. nNO is noninvasive, painless, easily performed from school age and has the capacity to discriminate between patients with PCD and healthy subjects in highly selected well-characterised populations [3-6]. To date, $\mathrm{nNO}$ has been reported to be very low, almost exclusively among subjects with PCD, and to separate convincingly between the presence and absence of PCD [3-8]. However, overlap in $\mathrm{nNO}$ values have been reported between PCD and other pulmonary diseases with symptoms mimicking PCD [9-11]. Hence, the true discriminative power of $\mathrm{nNO}$ in less selected, mixed population of patients referred for diagnostic testing of PCD will need to be settled before the role of $\mathrm{nNO}$ in the diagnosis of PCD can be fully appreciated.

Different techniques have been proposed, but not one technique fits all age groups. Recommendations for measurements have been provided [12], stressing the need for soft palate closure during sampling so as to avoid dilution from lower airways, but the standardisation of methodology is still lacking. Different manoeuvres during sampling have been evaluated [13-15], but most techniques are not applicable to infants and young children.

We hypothesised that $\mathrm{nNO}$ measurements, if adjusting sampling method according to age, could separate between patients with and without PCD in a real-life setting of consecutively referred patients in all age groups, however, with the expectation of a less convincing separation.

The aim of this study was to evaluate three different sampling methods for $\mathrm{nNO}$ measurements as first-line tests in a real-life setting of patients consecutively referred for PCD work-up, with the intention of expanding the age range for
AFFILIATIONS

Danish PCD Center, Paediatric

Pulmonary Service, Copenhagen University Hospital, Rigshospitalet, Copenhagen, Denmark.

CORRESPONDENCE

K.G. Nielsen

Paediatric Pulmonary Service, 5003, Copenhagen University Hospital Rigshospitalet Blegdamsvej 9 DK-2100 Copenhagen Denmark

E-mail: kgn@dadlnet.dk

Received:

March 012010

Accepted after revision: April 302010

First published online:

June 042010 
nNO measurements to include young children and infants with a view to achieving early PCD diagnosis.

\section{MATERIALS AND METHODS \\ Study subjects}

Four groups of subjects, children and adults, were included. 1) Patients with confirmed PCD from the National Danish PCD cohort [2] with consistent history and ongoing symptoms of PCD, and abnormal ciliary beat pattern and/or frequency (CBP and $\mathrm{CBF}$, respectively). Additionally, electron microscopy (EM) and measurements of pulmonary radioaerosol mucociliary clearance [16] had been performed in $78 \%$ and $68 \%$, respectively, of the patients. 2) Patients referred for PCD work-up. Patients with clinical symptoms and history suggestive of PCD consecutively referred from secondary paediatric and adult centres. Exclusion of cystic fibrosis (CF) and immunodeficiency was part of the initial work-up. 3) $\mathrm{CF}$ patients documented by sweat and genotype testing were recruited from the cohort of the CF Centre Copenhagen, Copenhagen, Denmark. 4) Healthy nonsmoking subjects (HS) were recruited among staff members and the children of staff.

All subjects gave their informed consent prior to participating in the study. The study was approved by the local ethics committee of The Capital Region of Denmark (KF 01-045/04).

\section{Study design}

The study consisted of three sub-studies. Sub-study one aimed to evaluate three different sampling methods for $\mathrm{nNO}$ measurements with the intention of expanding the age range for nNO measurements to include young children and infants. It was a cross-sectional study of children and adults with confirmed PCD or CF and healthy controls. Within occasion repeatability, long-term repeatability, acceptability, agreement between methods, dependency of age and subject category on repeatability and normative levels were assessed.

Sub-study two aimed to investigate the discriminative power of each of the three methods evaluated in sub-study one, determining sensitivity and specificity of cut-off values calculated to separate between presence and absence of PCD. It was a cross-sectional study of HS and selected patients with confirmed PCD.

Sub-study three aimed to test the three $\mathrm{nNO}$ sampling methods in a real-life setting in order to test their power to separate between PCD and non-PCD patients in a mixed cohort of patients with PCD-like symptoms consecutively referred for PCD work-up. It was a prospective study applying cut-off values from sub-study two. Final diagnostic work-up in terms of $\mathrm{CBP}, \mathrm{CBF}$ and $\mathrm{EM}$ was performed post hoc to $\mathrm{nNO}$ measurement.

\section{nNO measurement}

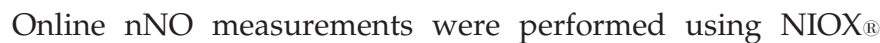
(Nitric Oxide Monitoring System; Aerocrine, Solna, Sweden) equipment. $\mathrm{nNO}$ gas was aspirated via a nasal olive probe inserted into one nostril by use of passive sampling flow rate of $5 \mathrm{~mL} \cdot \mathrm{s}^{-1}\left(\sim 0.3 \mathrm{~L} \cdot \mathrm{min}^{-1}\right)$, according to recommendations [12]. Prior to nNO measurement the subjects were interviewed about anatomical defects of the nose and sinuses. If the subject reported any known nasal defects from one side, the normal side was chosen. Three measurements were obtained at each session and the mean value was used. Outlying values, not further defined, were discarded at the discretion of the technician.

Measurements were postponed in cases of acute upper and lower airway infections within 2 weeks prior to $\mathrm{nNO}$ measurement. Any concurrent use of nasal decongestives or nasal steroids were unchanged during follow-up in sub-study one.

\section{Application of three sampling methods}

1) Breath hold (BH-nNO) sampling. This method assured soft palate closure and stable plateau values for at least $10 \mathrm{~s}$, in alignment with American Thoracic Society/European Respiratory Society (ERS) recommendations [12]. 2) Oral exhalation against resistance (OE-R-nNO). By use of a "party blow-out toy", temporary soft palate closure was accomplished during the active blowing [17]. The subjects were instructed to blow out in order to unfold the party blow-out toy and keep it inflated for as long as possible, thereby creating plateaus during 4-5 s of maximal nNO concentration. 3) Tidal breathing (TB-nNO). Normal relaxed tidal breathing was allowed during sampling. No soft palate closure is achieved by this method. Mean values were calculated from the highest three distinct visible peak concentrations, read directly as point values on the screen.

For each subject the choice of sampling method was adjusted according to age and level of cooperation. BH-nNO was not attempted in children $<4$ yrs of age. If cooperation with $\mathrm{BH}-$ $\mathrm{nNO}$ was anticipated in a given child, this was the first method of choice. If cooperation or technique then failed (i.e. breath hold was not possible for the child or no stable plateaus were seen), the $\mathrm{BH}-\mathrm{nNO}$ results were excluded and the next method in line, OE-R-nNO, was attempted. Again, if cooperation or technique failed (inability to blow the party blow-out toy sufficiently, or lack of plateaus) the results were omitted and the last method, TB-nNO, was performed. Thus, a formal acceptability study was not performed.

\section{Confirmative PCD diagnosis}

Diagnosis of PCD depended upon combined analysis of CBP and $\mathrm{CBF}$, and ultrastructure analysis as per the ERS Task Force consensus [1]. CBP and CBF were analysed in five to 10 nasal epithelial strips in a patient, using an interference contrast microscope (Leica DMLB; Leitz, Stuttgart, Germany) and digital frame-by-frame assessment of ciliary movements. EM pictures of $\geqslant 100$ ciliary cross-sections were evaluated for ultrastructural defects. Counts of outer and inner dynein arms were performed in a fraction of $\geqslant 10$ cross-sections.

\section{Analysis}

Median, range, mean and standard deviations were applied for baseline characteristics. Reliability of each sampling method was evaluated as coefficient of variation (CV) \% for all subjects. Repeatability between occasions was evaluated according to BLAND and ALTMAN [18] in a random sample of patients. Box and Whisker plots were used to demonstrate the separation between subject groups for each sampling method. In comparison of methods, limits of agreements (LoA) of OE-R$\mathrm{nNO}$ and TB-nNO were determined against $\mathrm{BH}-\mathrm{nNO}$ as the reference method. An unpaired t-test was used for comparison 
of mean $\mathrm{nNO}$ values between groups. The Wilcoxon rank sum test was applied when numbers were small. A p-value $<0.05$ defined the level of statistical significance.

\section{RESULTS}

In total, 282 subjects were included. Characteristics and distribution between subject groups are given in table 1. 117 patients were consecutively referred for PCD work-up of which $20(17.1 \%)$ were diagnosed with PCD. Ambient NO during measurements in 106 subjects was 19.9 (0.1-63.6) ppb.

\section{Sub-study one}

Normative data (mean \pm SE) ranged from $908 \pm 33$ ppb BH-nNO to $788 \pm 41 \mathrm{ppb}$ OE-R-nNO to $534 \pm 30 \mathrm{ppb}$ TB-nNO (figs 1-3).

Overall repeatability (CV\% (SD)) within occasion was $6.7 \%$ (8.8), $10.4 \%(14.2)$ and $12.3 \%$ (15.0) for BH-nNO, OE-R-nNO and TB-nNO, respectively. Among children aged $<6$ yrs, $\mathrm{CV} \%$ of TB-nNO was $10.8 \%$ (9.8) and thus age independent. Within occasion repeatability of $\mathrm{BH}-\mathrm{nNO}$ differed significantly between PCD $(9.7 \%)$ and HS $(3.6 \%)(p<0.0001)$, whereas no difference was shown between non-PCD referrals $(6.3 \%)$ and HS $(3.6 \%)$ or between referrals with PCD (10.6\%) and confirmed PCD patients $(9.7 \%)$. CV\% in CF was $4.8 \%$.

OE-R-nNO was in closer agreement with BH-nNO than TB$\mathrm{nNO}$. The $95 \%$ LoA between methods irrespective of diagnosis were 190.2 to $-166.9 \mathrm{ppb}$, mean \pm SD difference $11.7 \pm 178.5 \mathrm{ppb}$ (BH-nNO versus OE-R-nNO) and 572.2 to $-182.9 \mathrm{ppb}$, mean \pm SD difference $194.7 \pm 377.5 \mathrm{ppb}$ (BH-nNO versus TB-nNO).

Long-term repeatability assessed as mean $\mathrm{nNO}$ differences and LoA within variable time intervals (table A in the supplementary material) showed comparable $95 \%$ confidence intervals limits of all three methods.

Acceptability in children was judged from the proportion of subjects aged $<6$ yrs $(n=62)$ having performed each test successfully and the age range of such subjects. As such, the acceptability of TB-nNO was $95.2 \%$ with a minimum age of 14 days, thus highly exceeding acceptability of OE-R-nNO (25.8\%, minimum age of $2.5 \mathrm{yrs}$ ) and $\mathrm{BH}-\mathrm{nNO}(3.2 \%$, minimum age of $4.1 \mathrm{yrs})$.

\section{Sub-study two}

All methods discriminated significantly between PCD and HS (figs 1-3, table 2). However, unexpectedly high $\mathrm{nNO}$ values were seen in four $(6.8 \%)$ out of 59 of confirmed PCD patients, resulting in overlap between PCD and non-PCD.
Cut-off values with sensitivity/specificity/area under receiver operating characteristic curve providing the best discrimination between PCDs and HS were $175 \mathrm{ppb} / 91.1 / 100 / 0.95$ for BH-nNO, $242 \mathrm{ppb} / 94.3 / 100 / 0.97$ for OE-R-nNO, $158 \mathrm{ppb} /$ $94.4 / 100 / 0.97$ for TB-nNO and are further specified in the supplementary material table B and figs A, B and C.

nNO provided significant discrimination between PCD and CF patients in all methods (figs 1-3, table 2). However, despite significant discrimination, overlap independent of age occurred (figs 1-3, and figs. D, E and F in the supplementary material).

\section{Sub-study three}

Cut-off values from sub-study two were applied in the mixed cohort of 117 referrals (table $\mathrm{C}$ in the supplementary material), and the significant discrimination between PCD and non-PCD was completely mirrored in this unselected group of referrals. A solid diagnostic capacity was demonstrated by both $\mathrm{BH}-$ nNO and OE-R-nNO, and confirmed by high values of both sensitivity and specificity, whereas TB-nNO demonstrated poor specificity $(0.8)$ due to a high number $(n=17)$ of false positives (table $\mathrm{D}$ in the supplementary material).

Referrals without PCD and false positive TB-nNO were all children, median age (range) $2.7(0.2-6.3)$ yrs. The proportion of false positives was $39 \%$ in children aged $<6$ yrs, thereby correctly excluding $61 \%$ of referrals without PCD in this age group. None of these patients used nasal decongestives or nasal steroids.

During the study period we diagnosed PCD in two referred infants: a 16-day-old male with TB-nNO of $9 \mathrm{ppb}$ and an 8week-old female with TB-nNO of $11 \mathrm{ppb}$. One in 20 referrals with later confirmed PCD had above cut-off $\mathrm{nNO}$.

The overall number of false negatives $(n=5)$ among the PCD patients was substantial compared to previous studies. Clinical and diagnostic features of these patients with $\mathrm{nNO}$ values above cut-off are summarised in table 3 . None of these patients were atopic. Their fractional exhaled nitric oxide was normal (range 8-18 ppb).

\section{DISCUSSION}

This is the first large prospective study evaluating the usefulness of nNO measurement as a first-line test for PCD in an unselected cohort of PCD referrals with age range between infancy and adulthood. Our study confirmed earlier retrospective reports [3, 4, 7] in favour of $\mathrm{nNO}$ having a frontline role in the work-up for PCD. We examined three

\section{TABLE 1 Characteristics of included subjects}

\begin{tabular}{|c|c|c|c|c|c|}
\hline & Healthy subjects & Referrals for PCD work-up & PCD patients & CF patients & All subjects \\
\hline Subjects $\mathrm{n}$ & 57 & 117 & 59 & 49 & 282 \\
\hline Males & 19 (33.3) & $52(44.4)$ & $37(62.7)$ & $25(51.0)$ & $134(47.5)$ \\
\hline Age at inclusion yrs & $29.5(3.1-63.6)$ & $6.9(0.0-62.4)$ & $17.4(3.6-65.8)$ & $16.4(0.1-50.7)$ & $12.3(0.0-65.8)$ \\
\hline Age $<16$ yrs & $20(35.1)$ & $95(81.2)$ & $26(44.1)$ & $24(49.0)$ & $165(58.5)$ \\
\hline
\end{tabular}

Data are presented as $n(\%)$ or median (range), unless otherwise stated. PCD: primary ciliary dyskinesia; CF: cystic fibrosis 


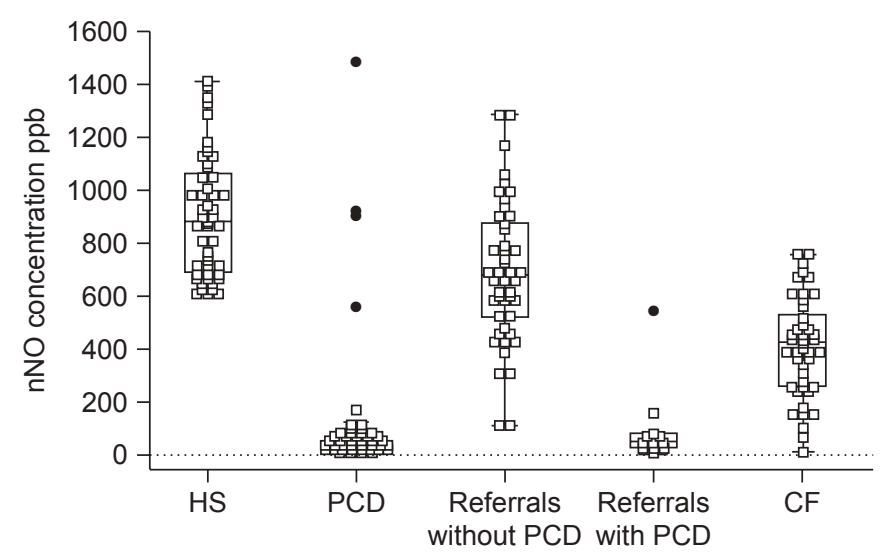

FIGURE 1. Box and Whisker plot. Breath hold nasal nitric oxide (nNO) concentrations in the subject categories: healthy nonsmoking subjects (HS), primary ciliary dyskinesia $(P C D)$, referrals with PCD, referrals without PCD and cystic fibrosis (CF). Dots and squares represent individual values. Dots designate far out values.

different sampling methods (BH-nNO, OE-R-nNO and TB$\mathrm{nNO}$ ), each intended for different age groups and levels of cooperation. All techniques demonstrated acceptable reliability, agreement and diagnostic capacity in addition to comparable long-term repeatability. This proves promising for the expansion of the age range of $\mathrm{nNO}$ measurements to include young children and infants. nNO levels in infant PCDs are so far largely uninvestigated and only published in a few case reports as extremely low [19-21]. We add to this literature two infants with TB-nNO values of $9 \mathrm{ppb}$ and $11 \mathrm{ppb}$, respectively.

Importantly, we report for the first time a remarkable number $(n=5)$ of PCD patients with high nNO levels (540-1486 ppb) and an incidence of above cut-off nNO levels reaching $6.8 \%$. High or normal nNO values in PCD is a phenomenon otherwise only very rarely reported [4,5]. Diagnosis of PCD may be difficult because secondary functional and structural abnormalities due to actual or recent infectious disease can be misinterpreted as primary disease [1]. In our study, all PCD patients demonstrating high $\mathrm{nNO}$ were re-evaluated for their clinical features and ciliary functional abnormalities. Furthermore, EM tests were repeated in three out of five cases and demonstrated identical ultrastructural abnormalities in the second test. All in all these patients were confirmed to be "true PCD patients," despite normal nNO. Clinical characteristics, as well as ciliary ultrastructural defects, varied greatly and did not give the impression of any common features. Notably, only one in five had situs inversus and none had chronic sinusitis. Whether absence of chronic sinusitis could be part of the explanation of high $\mathrm{nNO}$ in PCD remains to be shown, and further studies will be needed.

The attempt to evaluate nNO measurement in a real-life setting clarified some limitations regarding measurements in young children. TB-nNO could be measured without lower age limit, but led to progressive loss in power in terms of within-subject reliability and specificity. Thus, we report a considerable fraction of false positive cases among young children measured by TBnNO. Although $\mathrm{CV} \%$ was comparable in TB-nNO measured in children aged $<6$ yrs (10.8\%) and all age groups (12.3\%),

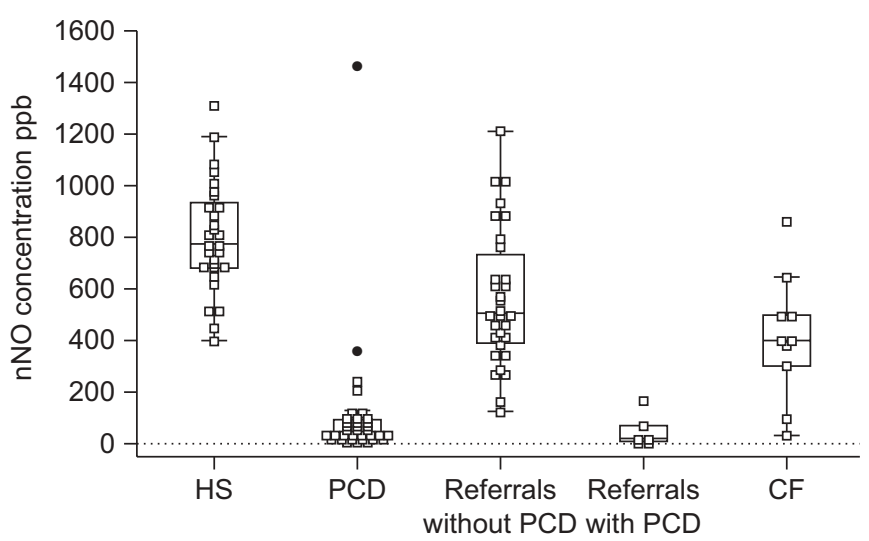

FIGURE 2. Box and Whisker plot. Oral exhalation against resistance nasal nitric oxide ( $\mathrm{nNO}$ ) concentrations in the subject categories: healthy nonsmoking subjects $(H S)$, primary ciliary dyskinesia (PCD), referrals with $P C D$, referrals without PCD and cystic fibrosis (CF). Dots and squares represent individual values. Dots designate far out values.

indicating that young children could repeat TB-nNO values as good as any individual, the overall $\mathrm{CV} \%$ of $\mathrm{TB}-\mathrm{nNO}$ was relatively high and agreement poor compared to BH-nNO.

TB-nNO is weakened by the lack of soft palate closure during sampling, where peak-values are read directly as single points on the screen, as opposed to better-defined plateaus provided by BH-nNO and OE-R-nNO sampling.

The high rate of false positive young children definitely represents a major weakness of TB-nNO. However, TB-nNO is a fast, easy, painless, noninvasive and well-accepted test in young children, and therefore has clear advantages compared to other tests involving either radiation [22] or high demands of cooperation [23]. TB-nNO also demonstrated a comparable negative predictive value (0.99) compared to $\mathrm{BH}-\mathrm{nNO}(0.98)$, and is thereby equally strong in ruling out PCD in cases with above cut-off values, e.g. we were able to exclude nearly twothirds of our non-PCD referrals aged $<6$ yrs by TB-nNO cutoff. TB-nNO is therefore suggested as a supplementary method

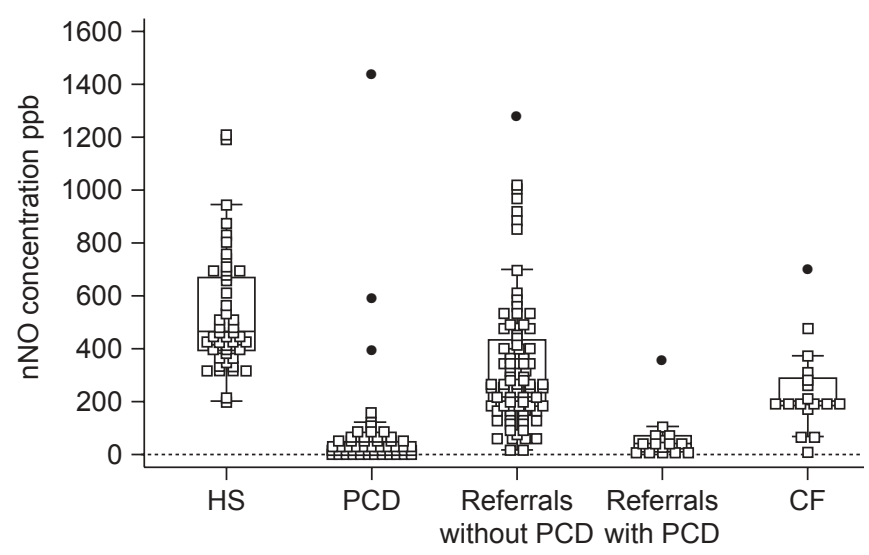

FIGURE 3. Box and Whisker plot. Tidal breathing nasal nitric oxide (nNO) concentrations in the subject categories: healthy nonsmoking subjects (HS), primary ciliary dyskinesia (PCD), referrals with PCD, referrals without PCD and cystic fibrosis (CF). Dots and squares represent individual values. Dots designate far out values. 


\begin{tabular}{|c|c|c|c|c|c|}
\hline \multirow[t]{2}{*}{ TABLE 2} & \multicolumn{5}{|c|}{$\begin{array}{l}\text { Comparison of nasal nitric oxide (nNO) levels } \\
\text { (ppb) between primary ciliary dyskinesia } \\
\text { (PCD), cystic fibrosis (CF) and healthy } \\
\text { nonsmoking subjects (HS) in the three sampling } \\
\text { methods }\end{array}$} \\
\hline & HS & PCD & CF & p-value ${ }^{\#}$ & p-value \\
\hline BH-nNO & $908 \pm 33(49)$ & $142 \pm 42(45)$ & $416 \pm 28(45)$ & $<0.0001$ & $<0.0001$ \\
\hline OE-R-nNO & $788 \pm 41(30)$ & $113 \pm 42(35)$ & $412 \pm 76(10)$ & $<0.0001$ & $<0.01$ \\
\hline TB-nNO & $534 \pm 30(52)$ & $86 \pm 28(54)$ & $243 \pm 39(17)$ & $<0.0001$ & $<0.01$ \\
\hline
\end{tabular}

in referrals not able to cooperate to either $\mathrm{BH}-\mathrm{nNO}$ or OE-RnNO. The expectation from TB-nNO should be to exclude a fraction of non-PCD referrals with above-cut off values from further investigation, with a view to minimise the amount of patients in need of further conventional ciliary function and ultrastructural analyses, which are both painful and overall time- and resource-consuming.

Normative nNO data in infants and young children are largely lacking as only very few studies exist, and contain very small groups of healthy children $[5,17,24]$. Specific reference values for this age group are important as non-PCD infants have also been shown to exhibit very low levels of $\mathrm{nNO}$, most probably caused by their undeveloped paranasal sinuses [19, 25, 26].

In this study, we present normative data in HS aged $>3$ yrs thereby probably setting cut-off values too high when applying these reference values to young children. Further studies are clearly needed to establish cut-off values for TB-nNO measurements in infants.

Previous studies in healthy subjects reported $\mathrm{CV} \%$ of repeated measurements to be dependent on age [24] and specific sampling technique [27]. The $\mathrm{CV} \%$ of $\mathrm{BH}-\mathrm{nNO}$ in a population of $\mathrm{HS}$ of all age groups was $3.6 \%$ and comparable with levels found in previous studies [24, 27]. However, in PCDs we found significantly higher $\mathrm{CV} \%$ probably explained by the low absolute values in PCD, which is in alignment with KARADAG et al. [4].

Acceptability of nNO measurements in children has only been addressed in few studies [20,28]. In one study, technical failure was found in $15 \%$ of 340 healthy school children [28]. A clear age-dependent acceptability of the three methods placed OER$\mathrm{nNO}$ as a useful technique to bridge the gap between methods regarding patients aged 2.5-6 yrs, who were noncompliant with $\mathrm{BH}$ technique. OER-nNO provides an opportunity to measure $\mathrm{nNO}$ while achieving plateaus during intermittent soft palate closure and thus providing more certain values compared to measurements with TB-nNO technique. From our data and experience we recommend the following order when attempting measurements in children aged $<6$ yrs: OE-R-nNO before TB-nNO.

A number of studies report low nNO levels in patients with an established diagnosis of PCD [3-7] including atypical PCD without typical ultrastructural defects characteristic of PCD

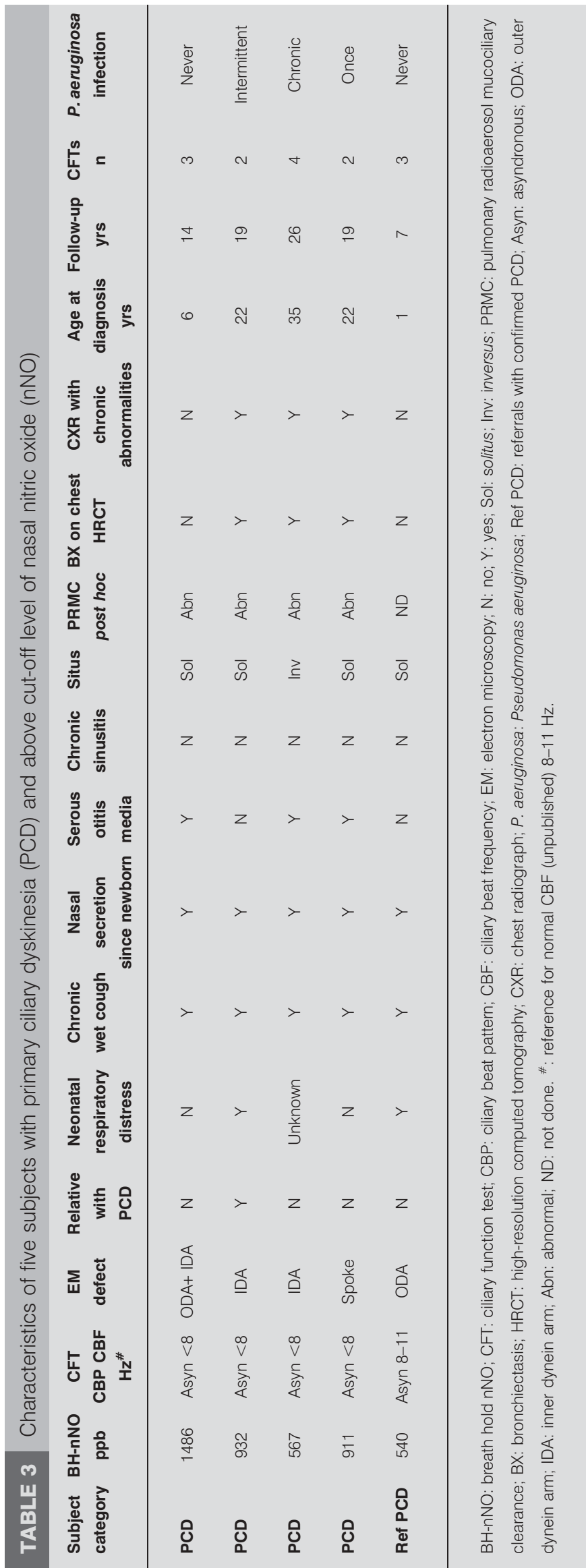


[8]. Additionally, $\mathrm{nNO}$ has been shown to be consistently low in PCD regardless of the use of different sampling techniques [29]. The reported cut-off levels and corresponding sensitivities/specificities varies between $105 \mathrm{ppb}$ and $94 \% / 88 \%$ [3], $187 \mathrm{ppb}$ and $93 \% / 95 \%$ [30], and $250 \mathrm{ppb}$ and $97 \% / 90 \%$ [5]. Differences may be explained by differences in nasal airflow during sampling and heterogeneity between study populations. In our study we took cut-off levels of 175 (BH-nNO), 242 (OE-RnNO) and 158 ppb (TB-nNO), and demonstrated comparable sensitivities for all three methods, whereas specificities were only comparable and acceptable for BH-nNO and OE-R-nNO. $\mathrm{BH}-\mathrm{nNO}$ was superior in subjects exhibiting full cooperation.

Discrimination between PCD and non-PCD was significant for all three methods, both between PCD and HS, and between PCD and non-PCD among the referred patients.

So far, results have been conflicting as to whether PCD and CF can be separated $[5,6]$ or cannot be separated [30] by nNO. In our study, $\mathrm{nNO}$ was found to discriminate significantly between PCD and CF irrespective of sampling method. However, overlap occurred unrelated to age, supporting the need to exclude CF before PCD work-up is initiated.

\section{Conclusion}

This is the first large study examining $\mathrm{nNO}$ as a first-line test for PCD in an unselected cohort of referrals of mixed age. We present normal reference values and cut-off values for three different nNO-sampling methods, and suggest an age-adjusted choice of $\mathrm{nNO}$ measurement in the following order: $\mathrm{BH}-$ nNO $>$ OE-R-nNO $>$ TB-nNO from adulthood through childhood into infancy. nNO discriminated highly significantly between PCD and non-PCD by all three methods, and OE-RnNO extended the age for soft palate closure measurements. TBnNO correctly excluded nearly two-thirds of non-PCD referrals aged $<6$ yrs. Future use of normative data from young children and infants may further strengthen the use of TB-nNO. Above cut-off $\mathrm{nNO}$ values were discovered in confirmed and consecutively referred PCDs, raising the concern that $\mathrm{nNO}$ can be misleading in a few patients with true PCD.

\section{SUPPORT STATEMENT}

$\mathrm{NIOX}_{\mathbb{B}}$ equipment was sponsored by The Lundbeck Foundation, Denmark.

\section{STATEMENT OF INTEREST}

A statement of interest for the present study can be found at www.erj. ersjournals.com/site/misc/statements.xhtml

\section{ACKNOWLEDGEMENTS}

We would like to thank M. Møller Andersen and M. Pejtersen (Danish PCD Center, Paediatric Pulmonary Service, Copenhagen University Hospital, Rigshospitalet, Copenhagen, Denmark), Biomedical Laboratory technologists for measuring $\mathrm{nNO}$ and performing ciliary function testing and N. Broholm (Dept of Pathology, Copenhagen University Hospital, Rigshospitalet) Biomedical Laboratory technologist for the EM preparation.

\section{REFERENCES}

1 Barbato A, Frischer T, Kuehni CE, et al. Primary ciliary dyskinesia: a consensus statement on diagnostic and treatment approaches in children. Eur Respir J 2009; 34: 1264-1276.
2 Marthin JK, Petersen N, Skovgaard LT, et al. Lung function in patients with primary ciliary dyskinesia. A cross sectional and three-decade longitudinal study. Am J Respir Crit Care Med 2010; 181: 1262-1268.

3 Corbelli R, Bringolf-Isler B, Amacher A, et al. Nasal nitric oxide measurements to screen children for primary ciliary dyskinesia. Chest 2004; 126: 1054-1059.

4 Karadag B, James AJ, Gultekin E, et al. Nasal and lower airway level of nitric oxide in children with primary ciliary dyskinesia. Eur Respir J 1999; 13: 1402-1405.

5 Narang I, Ersu R, Wilson NM, et al. Nitric oxide in chronic airway inflammation in children: diagnostic use and pathophysiological significance. Thorax 2002; 57: 586-589.

6 Wodehouse T, Kharitonov SA, Mackay IS, et al. Nasal nitric oxide measurements for the screening of primary ciliary dyskinesia. Eur Respir J 2003; 21: 43-47.

7 Lundberg JO, Weitzberg E, Nordvall SL, et al. Primarily nasal origin of exhaled nitric oxide and absence in Kartagener's syndrome. Eur Respir J 1994; 7: 1501-1504.

8 Pifferi M, Caramella D, Cangiotti AM, et al. Nasal nitric oxide in atypical primary ciliary dyskinesia. Chest 2007; 131: 870-873.

9 Lindberg S, Cervin A, Runer T. Nitric oxide (NO) production in the upper airways is decreased in chronic sinusitis. Acta Otolaryngol 1997; 117: 113-117.

10 Maniscalco M, Sofia M, Pelaia G. Nitric oxide in upper airways inflammatory diseases. Inflamm Res 2007; 56: 58-69.

11 Nakano $H$, Ide $H$, Imada $M$, et al. Reduced nasal nitric oxide in diffuse panbronchiolitis. Am J Respir Crit Care Med 2000; 162 2218-2220.

12 American Thoracic Society, European Respiratory Society. ATS/ ERS recommendations for standardized procedures for the online and offline measurement of exhaled lower respiratory nitric oxide and nasal nitric oxide, 2005. Am J Respir Crit Care Med 2005; 171 912-930.

13 Qian W, Djupesland PG, Chatkin JM, et al. Aspiration flow optimized for nasal nitric oxide measurement. Rhinology 1999; 37: 61-65.

14 Silkoff PE, Chatkin J, Qian W, et al. Nasal nitric oxide: a comparison of measurement techniques. Am J Rhinol 1999; 13: 169-178.

15 Weitzberg E, Lundberg JO. Humming greatly increases nasal nitric oxide. Am J Respir Crit Care Med 2002; 166: 144-145.

16 Marthin JK, Mortensen J, Pressler T, et al. Pulmonary radioaerosol mucociliary clearance in diagnosis of primary ciliary dyskinesia. Chest 2007; 132: 966-976.

17 Daya H, Qian W, McClean P, et al. Nasal nitric oxide in children: a novel measurement technique and normal values. Laryngoscope 2002; 112: 1831-1835.

18 Bland JM, Altman DG. Agreement between methods of measurement with multiple observations per individual. J Biopharm Stat 2007; 17: 571-582.

19 Baraldi E, Pasquale MF, Cangiotti AM, et al. Nasal nitric oxide is low early in life: case study of two infants with primary ciliary dyskinesia. Eur Respir J 2004; 24: 881-883.

20 Piacentini GL, Bodini A, Peroni D, et al. Nasal nitric oxide for early diagnosis of primary ciliary dyskinesia: practical issues in children. Respir Med 2008; 102: 541-547.

21 Stehling F, Roll C, Ratjen F, et al. Nasal nitric oxide to diagnose primary ciliary dyskinesia in newborns. Arch Dis Child Fetal Neonatal Ed 2006; 91: F233.

22 De Boeck C, Proesmans M, Mortelmans L, et al. Mucociliary transport using $99 \mathrm{mTc}$-albumin colloid: a reliable screening test for primary ciliary dyskinesia. Thorax 2005; 60: 414-417.

23 Bush A, Hogg C. Many a slip? Eur Respir J 2009; 34: 293-294.

24 Kharitonov SA, Walker L, Barnes PJ. Repeatability of standardised nasal nitric oxide measurements in healthy and asthmatic adults and children. Respir Med 2005; 99: 1105-1114. 
25 Chang CC, Lien SH, Liu TY, et al. Nasal nitric oxide in infants before and after extubation. Pediatr Pulmonol 2008; 43: 1072-1077.

26 Williams O, Rafferty GF, Hannam S, et al. Nasal and lower airway levels of nitric oxide in prematurely born infants. Early Hum Dev 2003; 72: 67-73.

27 de Winter-de Groot KM, van der Ent CK. Measurement of nasal nitric oxide: evaluation of six different sampling methods. Eur J Clin Invest 2009; 39: 72-77.
28 Struben VMD, Wieringa MH, Mantingh CJ, et al. Nasal NO: normal values in children age 6 through to 17 years. Eur Respir J 2005;26:453-457.

29 Santamaria F, De SS, Montella S, et al. Nasal nitric oxide assessment in primary ciliary dyskinesia using aspiration, exhalation, and humming. Med Sci Monit 2008; 14: CR80-CR85.

30 Horvath I, Loukides S, Wodehouse T, et al. Comparison of exhaled and nasal nitric oxide and exhaled carbon monoxide levels in bronchiectatic patients with and without primary ciliary dyskinesia. Thorax 2003; 58: 68-72. 